\title{
Medicolegal issues in abusive head trauma for the pediatric neurosurgeon
}

\author{
David I. Bass, MD, PhD, ${ }^{1}$ Amy Lee, MD,,2 Samuel R. Browd, MD, PhD, 1,2 \\ Richard G. Ellenbogen, MD, ${ }^{1,2}$ and Jason S. Hauptman, MD, PhD ${ }^{1,2}$

\begin{abstract}
'Department of Neurological Surgery, University of Washington; and 'Department of Neurosurgery, Seattle Children's Hospital, Seattle, Washington
\end{abstract}

\begin{abstract}
The purpose of this article is to serve as a rational guide for the pediatric neurosurgeon in navigating common medicolegal issues that arise in the management of abusive head trauma (AHT). Many of these issues may be unfamiliar or unpleasant to surgeons focused on addressing disease. The authors begin with a brief history on the origins of the diagnosis of AHT and the controversy surrounding it, highlighting some of the facets of the diagnosis that make it particularly unique in pediatric neurosurgery. They then review some special medical considerations in these patients through the perspective of the neurosurgeon and provide several examples as illustration. The authors discuss how to appropriately document these cases in the medical record for expected legal review, and last, they provide an overview of the legal process through which the neurosurgeon may be called to provide testimony.
\end{abstract}

https://thejns.org/doi/abs/10.3171/2020.8.FOCUS20599

KEYWORDS medicolegal; abusive head trauma; legal documentation

A BUSIVE head trauma (AHT) is a medicolegal diagnosis based on medical information presented by healthcare providers and determined by the judgment of legal professionals and jurors. The diagnosis not only indicates mechanism of action but also intention. As such, it is a unique diagnosis that presents the neurosurgeon with many unfamiliar challenges. It is unfortunately common in the pediatric population, with an incidence between 13 and 40.5 per 100,000 per year in infants younger than 1 year of age, and nearly a quarter of those cases result in the death of the child. ${ }^{1,2}$ Thus, a diagnosis of AHT may progress to a legal definition of homicide in select cases.

The purpose of this article is to familiarize the pediatric neurosurgeon with some of the more common medicolegal issues surrounding AHT as well as to discuss legal commitments and ethical obligations of the neurosurgeon in these cases.

\section{A History of Controversy}

The history of AHT is fraught with medical and legal controversy, particularly with regard to shaken baby syndrome (SBS). Descriptions of injuries that raise suspicion of child abuse were first documented in the middle of the 19th century. ${ }^{3,4}$ In 1946, a radiologist named Dr. John Caffey published an article associating subdural hematomas with specific patterns of bone injuries in the pediatric population..$^{5}$ In 1972, he postulated that these injuries were linked by a common mechanism, specifically whiplash sustained during violent shaking. ${ }^{6} \mathrm{He}$ also noted the frequency of retinal hemorrhages in these children, and these observations led to the conclusion that the SBS triad (bilateral subdural hematomas, retinal hemorrhages, and encephalopathy) was pathognomonic for the diagnosis of AHT. ${ }^{7}$ However, the hypothesis that shaking a small child could cause this triad of injuries was developed before the era of evidence-based medicine, and therefore many studies on the topic prior to 1998 lacked the statistical rigor of today's standards. ${ }^{8}$ As such, the diagnosis of AHT, and particularly that of SBS, came under intense scrutiny and was criticized as a diagnosis predicated on flawed science. ${ }^{3}$ Legal defenses were able to exploit these weaknesses and used the medical complexity of AHT to confuse juries into doubting the validity of AHT as a medical diagnosis. ${ }^{9}$ This scrutiny in part led to the surge in appeals of criminal convictions for SBS seen in the 1990s and early 2000s. ${ }^{7}$ 
Importantly, a realization emerged that the pattern of injuries seen with SBS, although highly suggestive of an inflicted injury, is not sufficiently unique to be considered pathognomonic for SBS. ${ }^{2}$ Some have argued that the SBS triad was developed as a legal tool for the courtroom rather than as a reflection of a true clinical syndrome..$^{10}$ Indeed, there is no gold standard for the diagnosis of SBS or AHT, but many efforts have been made to develop tools that can accurately identify when abuse has occurred based on the pattern of injuries. ${ }^{11}$ The need for such a tool stems from the fact that these events are seldom witnessed and the perpetrator does not confess to the crime in most cases. ${ }^{12}$ Adding further to the confusion, unsubstantiated theories, such as dysphagic choking, ${ }^{13}$ have been developed for use in the courtroom as alternative explanations for these injuries, with minimal scientific or medical merit. ${ }^{14}$ These strategies underscore the fact that much of the legal controversy surrounding SBS has largely been fabricated..$^{10,15}$ Regardless, the medical community has moved away from use of the term "SBS" and toward the term "AHT," which does not specify the mechanism of injury.

There is a broad medical consensus that AHT causes injuries seen in the SBS triad as well as other characteristic injuries, but there has been much debate over the precise mechanism through which the injuries are inflicted. The presumption of shaking was based on both confessions from perpetrators and the fact that many children had no external signs of injury. ${ }^{16}$ However, biomechanical studies in adult primates have suggested that the forces generated by shaking are insufficient to cause the injuries seen in children with AHT. ${ }^{17}$ In contrast, the forces generated by abrupt deceleration from striking a hard object may be 50 times greater. Further, Duhaime et al. reported that signs of blunt trauma could be found in most children diagnosed with SBS and, upon autopsy, in all children who died from SBS. ${ }^{18}$ They argued that signs of external trauma may not be as visible in babies because their skulls are much more pliable than adult skulls, thereby distributing the force of impact over a broader surface area. However, other researchers have criticized the biomechanical studies, arguing that adult primates do not accurately model the tissue properties of an infant's head and neck and that the single-cycle rotation used to inflict injury in these studies does not reflect the biomechanical forces generated during violent shaking. ${ }^{16}$

Since these studies were first performed, other groups have attempted to replicate the distinctive injury patterns of AHT by shaking juvenile animal models such as piglets and lambs. They found that younger animals with biomechanical tissue properties more akin to those found in infants are more susceptible to traumatic brain injuries from shaking and that multiple injuries inflicted over short intervals can compound and progress with time..$^{19,20}$ Despite these findings, the injuries sustained from these simulations remained relatively minor compared to those observed with AHT. Indeed, hypoxic injury, the most common neuropathological feature of AHT, ${ }^{21}$ was observed in only a small minority of animal subjects. Such discrepancies have been attributed to using anesthetized animals and animals with more mature neuroanatomy.

Some of the difficulty in modeling AHT stems from the dearth of direct data (e.g., video of the incident) on which the precise mechanisms can be reproduced. As a result, efforts to better understand AHT through isolating cranial injury may miss critical components of AHT pathophysiology such as injuries to the craniocervical junction. ${ }^{20}$ Injuries to the nuchal ligaments can be found in up to $78 \%$ of children with AHT, and it has been hypothesized that hyperflexion injuries at the cervicomedullary junction may cause a transient neuropraxia that presents as an apneic event. ${ }^{1,22}$ It is important to note that, despite this academic debate, there is a general consensus that violently shaking a small child is likely to inflict harm.

\section{Medical Obligations of the Neurosurgeon in Cases of Suspected AHT}

A key component in the workup for AHT is the development and thoughtful evaluation of a thorough differential diagnosis. Most alternative explanations broadly fall under two categories, accidental trauma and medical/ congenital abnormalities, with accidental trauma being the more common of the two..$^{23}$

Nonaccidental trauma is a common mechanism of severe injury in children, and thus providers should consider AHT when head injuries are identified. ${ }^{4}$ Compared to head injuries from accidental trauma, AHT trauma is more common in younger children, especially those younger than 4 months of age, and it tends to lead to more severe presentations. ${ }^{24}$ Specifically, bilateral subdural hematomas, interhemispheric subdural hematomas, retinal hemorrhages, seizures, apnea, impaired consciousness, diffuse cerebral edema, hydrocephalus, complex skull fractures, and bruising of the torso, ears, and neck are more common in cases of AHT, while simple parietal skull fractures and epidural hematomas are more common in cases of accidental trauma. ${ }^{1,11,24}$ There are special examples of accidental trauma, such as falling down stairs, that can present with severe injuries in patterns similar to AHT, but these are typically associated with external signs of trauma. ${ }^{1}$ A traumatic birth can cause bilateral subdural hematomas and retinal hemorrhages, but these injuries tend to be asymptomatic and resolve after 4 weeks.

Various coagulopathies, metabolic disorders, and congenital abnormalities can present similarly to AHT. Perhaps one of the more common imitators is macrocephaly from external hydrocephalus. ${ }^{1}$ In this condition, enlargement of the subarachnoid spaces may be confused for chronic, bilateral subdural hematomas. This condition is benign and needs no further management. Children with glutaric aciduria, a rare genetic disorder, can also present with macrocephaly. They are prone to develop bilateral subdural hematomas and retinal hemorrhages. Basal ganglia necrosis and open sylvian fissures seen on cranial imaging can help distinguish this metabolic disease from AHT. These examples illustrate the importance of a thorough workup to rule out underlying genetic defects and other coagulopathies in the evaluation of AHT.

In general, injuries sustained from AHT are more likely to require neurosurgical intervention, ${ }^{24}$ and therefore the neurosurgeon plays a critical role in the evaluation and treatment of children with AHT. Indeed, the neurosurgeon 
has a special obligation to self-educate on the management of AHT and on how to distinguish AHT from accidental traumatic injury and other medical conditions. The neurosurgeon should help guide the diagnostic workup for AHT, and he or she is responsible for carefully documenting historical reports, physical examination findings, and radiographic interpretations. Furthermore, the neurosurgeon is often the sole provider who relays intraoperative findings, such as injuries identified upon reflection of the scalp, that can help determine the mechanism of injury. As such, these findings must be clearly documented in the medical records. Nevertheless, the neurosurgeon need not speculate as to the mechanism of injury, and in general, is advised against doing so.

\section{Legal Commitments of the Neurosurgeon in Cases of Suspected AHT}

Many neurosurgeons find that the legal process surrounding AHT is cumbersome, unfamiliar territory. Determinations of AHT can interrupt clinical practices and throw the neurosurgeon into an uncomfortable, adversarial environment. However, it is important to remember that the role of the neurosurgeon in the legal process is as an advocate for the truth, not necessarily an advocate for justice for a particular side. The ability to advocate for the truth depends on having a good foundation of knowledge regarding AHT and on thorough documentation of the case. Medical records are legal documents. These legal cases may continue for 2 or 3 years as they laboriously navigate the judicial system. This interval can make it difficult to remember the precise details of a case, unless details are written down. Furthermore, documentation must be approached with the understanding that AHT is not an ordinary brain injury. Details of the case should be documented with a mechanism in mind, but, once again, speculation and editorials on antecedent events should be avoided.

The legal process itself varies substantially from case to case and location to location. The process outlined below is a generalization based on cumulative experiences with the Safe Child and Adolescent Network at Seattle Children's Hospital.

AHT cases are typically handled by the office of the state's attorney general. If a neurosurgeon is to be involved, the office may contact him or her via the legal service office with a subpoena for deposition testimony. This subpoena mandates the neurosurgeon to meet with a court reporter and attorneys from both the prosecution and the defense, but the neurosurgeon can request that this meeting happen after normal business hours so as not to interrupt clinical practice. The neurosurgeon can be called as either a fact witness or an expert witness, although the state cannot compel the treating physician to serve as an expert witness in the technical sense of the term. The role of the fact witness can be viewed as an extension of the patient-surgeon relationship. The interview will focus on the specific findings identified by the neurosurgeon and the details of the care that was provided. In contrast, an expert witness will offer opinions on what might have happened and answer hypothetical questions. The expert witness will be asked to determine whether abuse occurred but should avoid weighing in on a verdict as this is determined by the judge or jury. Notably, the verdict tends to rely heavily on the debate between expert witnesses from opposing sides. In general, neurosurgeons are discouraged to serve as both a fact witness and an expert witness, because the latter may discredit the testimony of the former.

Once subpoenaed, the neurosurgeon should contact their institution's risk management office. Risk management may provide legal resources and advice, and prevent the provider from becoming a subject of scrutiny in the case. The attorney general's office should be contacted following consultation with risk management to schedule the deposition. The deposition typically lasts several hours and covers questions from both legal teams. An expert witness is more likely to encounter an adversarial environment in which his or her credibility may be challenged, but regardless of the neurosurgeon's role, the provider is expected to render expert medical testimony based on "reasonable medical certainty." It is important to understand that there is no legal consensus on the definition of this term. In fact, the term is fluid and appears to vary quite widely between the different experts who are testifying and their levels of expertise. ${ }^{25}$

Most cases enter a plea deal, settle, or are dropped for procedural matters, but a small minority of cases, perhaps $5 \%$, will go to trial. If the case goes to trial, the neurosurgeon may receive a second subpoena for trial testimony. In general, there are two types of trials, civil (or family) and criminal. Civil proceedings determine custody of the child, and the evidentiary standard upon which decisions are made varies among states from "preponderance of evidence" to "clear and convincing." In contrast, criminal proceedings determine guilt, and the verdict is rendered based on the standard of "beyond a reasonable doubt." Although it is important to know the functional differences between types of proceedings, the actual role of the neurosurgeon does not vary. Indeed, all testimony should be as precise and factual as possible and delivered within a reasonable degree of medical certainty. When uncertainty emerges during a line of questioning, the neurosurgeon should state that uncertainty exists. A strictly "yes" or "no" response is neither required nor appropriate in such circumstances.

\section{Conclusions}

AHT is an all too common occurrence within the pediatric population. It is particularly unique among medical diagnoses because of the legal implications imposed by the diagnosis, and therefore it has been the subject of much legal controversy over the decades. As the neurosurgeon possesses an exceptional expertise that is particularly relevant to treating these patients, he or she plays a critical role in these cases as an advocate for the truth. This role necessitates that the neurosurgeon is well versed on the presentation of AHT and on scientific controversies surrounding the diagnosis. The neurosurgeon should be capable of performing a thorough workup for evaluating the differential diagnosis in AHT, while providing precise and comprehensive documentation of his or her findings. 
In the event of a subpoena for testimony, the neurosurgeon should approach his or her institution for guidance and access to legal resources.

\section{Acknowledgments}

We thank Mark S. Dias, MD, Penn State Health, Hershey, PA, and Caitlin Morray, JD, Director of Risk Management at Seattle Children's Hospital, Seattle, WA, for their support and substantive input on this publication. The authors appreciate the assistance of Sharon Durfy, PhD, with manuscript preparation.

\section{References}

1. Boos SC, Dias MS. Abusive head trauma. In: Jallo G, Kothbauer K, Recinos V, eds. Handbook of Pediatric Neurosurgery. Thieme Medical Publishers; 2018.

2. Narang SK, Fingarson A, Lukefahr J. Abusive head trauma in infants and children. Pediatrics. 2020;145(4):e20200203.

3. Narang SK. A Daubert analysis of abusive head trauma/ shaken baby syndrome. Houst J Health Law Policy. 2012;11: 505-633.

4. Paul AR, Adamo MA. Non-accidental trauma in pediatric patients: a review of epidemiology, pathophysiology, diagnosis and treatment. Transl Pediatr. 2014;3(3):195-207.

5. Caffey J. Multiple fractures in the long bones of infants suffering from chronic subdural hematoma. Am J Roentgenol Radium Ther. 1946;56(2):163-173.

6. Caffey J. On the theory and practice of shaking infants. Its potential residual effects of permanent brain damage and mental retardation. Am J Dis Child. 1972;124(2):161-169.

7. Tuerkheimer D. The next Innocence Project: shaken baby syndrome and the criminal courts. Wash Univ Law Rev. 2009;87(1):1-58.

8. Donohoe M. Evidence-based medicine and shaken baby syndrome: part I: literature review, 1966-1998. Am J Forensic Med Pathol. 2003;24(3):239-242.

9. Albert DM, Blanchard JW, Knox BL. Ensuring appropriate expert testimony for cases involving the "shaken baby." JAMA. 2012;308(1):39-40.

10. Greeley CS. "Shaken baby syndrome" and forensic pathology. Forensic Sci Med Pathol. 2014;10(2):253-255.

11. Hymel KP, Wang M, Chinchilli VM, et al. Estimating the probability of abusive head trauma after abuse evaluation. Child Abuse Negl. 2019;88:266-274.

12. Adamsbaum C, Grabar S, Mejean N, Rey-Salmon C. Abusive head trauma: judicial admissions highlight violent and repetitive shaking. Pediatrics. 2010;126(3):546-555.

13. Barnes PD, Galaznik J, Gardner H, Shuman M. Infant acute life-threatening event-dysphagic choking versus nonaccidental injury. Semin Pediatr Neurol. 2010;17(1):7-11.

14. Edwards GA. Mimics of child abuse: Can choking explain abusive head trauma? J Forensic Leg Med. 2015;35:33-37.

15. Moreno JA, Holmgren B. Dissent into confusion: the supreme court, denialism, and the false "scientific" controversy over shaken baby syndrome. Utah Law Rev. 2013;13:153.
16. Dias MS. The case for shaking. In: Carole J, ed. Child Abuse and Neglect: Diagnosis, Treatment and Evidence. Elsevier Saunders; 2010:362-371.

17. Duhaime AC, Christian CW, Rorke LB, Zimmerman RA. Nonaccidental head injury in infants-the "shaken-baby syndrome." N Engl J Med. 1998;338(25):1822-1829.

18. Duhaime AC, Gennarelli TA, Thibault LE, et al. The shaken baby syndrome. A clinical, pathological, and biomechanical study. J Neurosurg. 1987;66(3):409-415.

19. Finnie JW, Blumbergs PC, Manavis J, et al. Neuropathological changes in a lamb model of non-accidental head injury (the shaken baby syndrome). J Clin Neurosci. 2012;19(8): 1159-1164.

20. Coats B, Binenbaum G, Smith C, et al. Cyclic head rotations produce modest brain injury in infant piglets. $J$ Neurotrauma. 2017;34(1):235-247.

21. Geddes JF, Hackshaw AK, Vowles GH, et al. Neuropathology of inflicted head injury in children. I. Patterns of brain damage. Brain. 2001;124(Pt 7):1290-1298.

22. Choudhary AK, Ishak R, Zacharia TT, Dias MS. Imaging of spinal injury in abusive head trauma: a retrospective study. Pediatr Radiol. 2014;44(9):1130-1140.

23. Duhaime AC, Christian CW. Abusive head trauma: evidence, obfuscation, and informed management. J Neurosurg Pediatr. 2019;24(5):481-488.

24. Adamo MA, Drazin D, Smith C, Waldman JB. Comparison of accidental and nonaccidental traumatic brain injuries in infants and toddlers: demographics, neurosurgical interventions, and outcomes. J Neurosurg Pediatr. 2009;4(5): 414-419.

25. Dias MS, Boehmer S, Johnston-Walsh L, Levi BH. Defining 'reasonable medical certainty' in court: what does it mean to medical experts in child abuse cases? Child Abuse Negl. 2015;50:218-227.

\section{Disclosures}

Dr. Hauptman is a consultant for Medtronic.

\section{Author Contributions}

Conception and design: Hauptman, Lee. Acquisition of data: Bass. Analysis and interpretation of data: Hauptman, Bass, Lee. Drafting the article: Bass. Critically revising the article: Hauptman, Bass, Lee, Ellenbogen. Reviewed submitted version of manuscript: all authors. Approved the final version of the manuscript on behalf of all authors: Hauptman. Administrative/ technical/material support: Hauptman, Lee. Study supervision: Hauptman.

\section{Correspondence}

Jason S. Hauptman: Seattle Children's Hospital, Seattle, WA. respub@uw.edu. 